\title{
EL CONFLICTO LABORAL EN LA ACTUALIDAD: LOS NUEVOS CONFLICTOS*
}

Francisco Pérez de los Cobos Orihuel**

Voy a hablarles de la evolución del conflicto y de los nuevos conflictos, es decir, de los cambios operados en el sistema de relaciones laborales y de las repercusiones que de esos cambios derivan para la morfología del conflicto industrial, tal y como éste ha venido siendo concebido, y de los nuevos conflictos, que están apareciendo como consecuencia de las transformaciones económicas y sociales.

Las transformaciones económicas, productivas y sociales que están determinando los nuevos perfiles del conflicto son múltiples y de diverso cuño. En aras de la claridad expositiva, las agruparé, en función del escenario en el que se verifican, en tres grupos: aquellas que afectan al sistema económico en su conjunto, aquellas que afectan a la morfología y organización de la empresa, y las que se refieren al trabajo mismo, a su nueva composición y a sus nuevos sistemas de organización.

\section{El CONFlicto ante LOS CAmbios en el SISTEMA ECONÓmico}

El primer y fundamental cambio económico que está afectando profundamente nuestros sistemas de relaciones de trabajo y configurando un "nuevo conflicto industrial" es la "globalización". La extensión planetaria del dominio del mercado, la creación de un mercado de envergadura mundial afecta, desde luego, en primer lugar, a las empresas que quedan ahora sometidas a una concurrencia global y deben organizar la producción de bienes y servicios a escala planetaria, pero en no menor medida interesa a los trabajadores y a sus organizaciones representativas por cuanto la globalización prefigura una nueva "división internacional del trabajo" y obliga al sindicato a pensar a escala global y a internacionalizarse. No descubro nada a nadie si les digo que en muchos sectores productivos, sobre todo en el sector servicios (transportes, comunica-

\footnotetext{
* Conferencia presentada en las X Jornadas de la Fundación Servicio Interconfederal de Mediación y Arbitraje, Madrid, 25 de septiembre del 2008. Colaboración recibida el 5 de abril y aprobada el 16 de abril de 2010.

** Catedrático de Derecho del Trabajo y de la Seguridad Social de la Universidad Complutense de Madrid. Correo electrónico: franciscoperezdeloscobos@der.ucm.es.
} 
ciones, energía, banca, etc.), tanto la propiedad como la gestión empresarial misma, trascienden claramente las dimensiones nacionales.

El desarrollo de los mercados financieros, la introducción masiva de las tecnologías de la información y de las telecomunicaciones, el abaratamiento de los costes de transportes y de las comunicaciones han hecho factible un espectacular aumento de la movilidad del capital, y consecuencia de ello, el que los procesos de deslocalización y relocalización productiva, a nivel regional y mundial, se multipliquen y adquieran visos de normalidad. Estos procesos en Europa afectan, en primer término, a las industrias de trabajo intensivas, respecto de las cuales los costos de producción, señaladamente el costo de la mano de obra, son un factor determinante de la competitividad. Pero, como ha alertado la Comisión Europea, "comienzan igualmente a observarse en sectores intermedios, que constituyen los puntos fuertes tradicionales de la industria europea, e incluso en ciertos sectores de alta tecnología en los que existen indicios de deslocalización de ciertas actividades de investigación y en los servicios".

Al decir de los expertos, la economía española es particularmente vulnerable al proceso de deslocalización, "ad intra", en el seno de la Unión Europea con relación a los PECOS, y ad extra, frente a los nuevos países industrializados. Nuestro modelo de desarrollo y las debilidades de nuestro sistema productivo, nuestra deficiente especialización productiva hacen que, para nosotros, la concurrencia entre territorios para atraer inversiones, para evitar o atraer deslocalizaciones estén a la orden del día.

La globalización, como es obvio, internacionaliza los conflictos de trabajo y obliga a los actores a pensar y a actuar desde este nuevo escenario. Comoquiera que la empresa o el grupo de empresas concibe y planifica sus actuaciones globalmente, el conflicto se internacionaliza y el sindicato, desbordado por la nueva dimensión de los problemas, reformula sus modos de actuación.

Las experiencias españolas de deslocalización evidencian la dimensión internacional de estos nuevos conflictos que está omnipresente a la hora de elaborar las estrategias para abordarlos. Simplificando las cosas pero sin faltar a la verdad pueden identificarse en los procesos de deslocalización operados en nuestro país en los últimos años, sobre todo en Cataluña, dos grandes estrategias empresariales. En primer lugar, la de evitar el cierre de la factoría mediante la renegociación de las condiciones de trabajo aplicables en la empresa y el establecimiento de medidas de mejora de la productividad, a cambio de la garantía del mantenimiento de la inversión y el empleo e incluso el aumento de aquélla y de éste. En la base de esta estrategia está la convicción de que la única vía para garantizar la viabilidad de la empresa es la restauración de su competitividad, mejorando la productividad, por eso estos pactos suelen versar sobre las condiciones de trabajo más ligadas a la productividad de la empresa, esto es, la jornada y los salarios. Básicamente se trata de extender la jornada 
manteniendo el salario inalterado o de rebajar el salario y menguar con ello el costo de trabajo para la empresa.

La segunda estrategia, que se acomete a veces cuando fracasa la primera -el caso de Philips es, en este sentido, paradigmático- y otras directamente, es la del cierre de la empresa a través normalmente de la regulación del empleo. También en la base de estas decisiones de cierre hay una clara valoración de la dimensión internacional del problema: la empresa cierra aquí porque la competencia ya produce allí a costos muy inferiores o porque quiere mejorar su competitividad y adelantarse a la competencia.

Pues bien, el marco institucional del que disponemos para afrontar esta nueva conflictividad derivada de los procesos de deslocalización presenta claras insuficiencias. Por lo que se refiere a las primeras estrategias empresariales aludidas, que se han traducido en la práctica en la incorporación a la negociación colectiva de dobles escalas salariales, han topado con la rigurosa aplicación que nuestra jurisprudencia hace del principio de la igualdad en la ley a los convenios colectivos estatutarios, que priva al convenio de la posibilidad de matizar o programar su aplicación en la empresa en atención a otros contenidos negociales. El único supuesto en el que es posible instrumentar una escala salarial diversificada en convenio estatutario con plenas garantías de que no pueda anularse por considerarla atentatoria contra el principio de igualdad es la doble escala salarial con carácter transitorio. La aplicación del principio de igualdad es para el convenio colectivo "res extra comercium", lo que penaliza sin duda el importantísimo papel de "gestión de las relaciones de trabajo" que hoy corresponde desempeñar a la autonomía colectiva. Los únicos expedientes que, al parecer, no plantearían problemas para instrumentar estos pactos de mejora de la productividad serían el descuelgue salarial y la modificación "ante tempus" del convenio colectivo, vías ambas en las que resulta muy factible la utilización de procedimientos extrajudiciales de solución de conflictos.

Las insuficiencias del art. 51 ET para abordar los procesos de deslocalización productiva derivan básicamente del hecho de que dicho precepto, que concibe el despido económico como un instrumento de restauración de la vialidad empresarial, se aplica por voluntad expresa del legislador al cierre de la empresa. En efecto, tanto la formulación legal de las causas para despedir, como el procedimiento de regulación de empleo, como, en fin, la intervención de la Administración en el expediente administrativo no se adecuan a la realidad del cierre empresarial del establecimiento, por eso hoy por hoy el conflicto derivado de la decisión del cierre se focaliza en la determinación del montante de las indemnizaciones que percibirán los despedidos, a los que se les tutela desde el contrato de trabajo cuando se los expulsa al mercado de trabajo. A mi juicio, éste debería ser un ámbito de desarrollo de los sistemas extrajudiciales de conflicto, pero para que tal desarrollo se produzca probablemente es necesaria 
una regulación del despido por cierre menos burda de la que tenemos. Pero lo burdo puede resultar cómodo, caro quizá, pero cómodo: pago y escapo, y en ésas estamos.

El segundo cambio económico que está determinando la transformación del conflicto laboral es la pérdida de peso de la industria en el conjunto del sistema económico a favor del sector servicios. El conflicto laboral se ha hecho en la industria - no en balde se le llama conflicto industrial-y la cultura del conflicto es, principalmente, una cultura industrial. Pero la industria no ha hecho más que perder peso en el conjunto de la economía y del empleo y presumiblemente tal será su evolución futura. Desde los años sesenta, las actividades industriales en los países de la OCDE y, en paralelo, el volumen de empleo industrial no han hecho sino disminuir. Si en los sesenta las actividades industriales suponían el $30 \%$ del PIB de los países de la OCDE, en 1995 suponían ya el $20 \%$ y desde entonces no ha hecho más que decrecer. El empleo industrial, por su parte, que en 1970 era del $28 \%$, en 1995 era ya del $17 \%$. Para algunos analistas es muy probable que en pocas décadas el empleo industrial en la OCDE caiga por debajo del $10 \%$, porque enteros sectores de la industria, y en primer lugar los más intensivos en mano de obra, se deslocalizan.

Una industria que será -lo está siendo ya- mucho más "terciarizada" que la actual, pues cada día necesita más servicios para llevar a cabo su propia actividad productiva, tanto servicios tradicionales como avanzados.

Pues bien, la "terciarización" de la economía está transformando sustancialmente la morfología del conflicto laboral. La "terciarización" económica es la del conflicto mismo en un doble sentido: se produce, en primer lugar, una "deslocalización" del conflicto hacia el sector terciario, donde éste, por su repercusión pública, se revela más eficaz que la industria; en segundo lugar, en parte como consecuencia de la "terciarización" de la economía y en parte por los cambios que de ella han derivado para la propia morfología de conflicto "terceros", esto es, consumidores y usuarios, se ven comprometidos en el conflicto y utilizados como rehenes en las viejas y nuevas formas de acción colectiva. El conflicto es hoy más eficaz en la medida en la que a su lesividad económica añade una lesividad social, en la medida en la que afecta a la ciudadanía y adquiere dimensión pública. Se trata, con todo, de un arma de doble filo, pues lo que resulta para la acción colectiva un instrumento de poder, puede serlo también de deslegitimación social.

Quizá por ello, hoy como nunca, los sindicatos son conscientes de la necesidad de gobernar el conflicto y temperar o impedir su lesividad social. Pues bien, los procedimientos de solución extrajudicial de conflictos podrían utilizarse como instrumento regulador en frío del propio recurso al conflicto y de su incidencia sobre los servicios esenciales para la Comunidad. Se trataría de establecer códigos de regulación del conflicto, de suerte que cuando la situación 
colectiva se produjese, el conflicto se atuviera a lo pactado y menguara así su lesividad social. En otros países, por ejemplo en Italia, son ya una realidad en un sistema de relaciones laborales y están contribuyendo tanto a gobernar el conflicto y temperar sus consecuencias como a legitimar a los sindicatos que los suscriben, que así pueden acreditar ante la opinión pública que hacen un uso responsable de la huelga. El establecimiento "a priori" de un preaviso que advierta a los consumidores y usuarios con antelación suficiente de la huelga y la elección de los días de huelga y de las modalidades de huelga menos lesivas para la organización social de las medidas aparentemente simples -su articulación normativa es siempre compleja- pero que contribuyen decisivamente a garantizar el ejercicio de la huelga y su tolerabilidad social. Como les decía estos códigos están siendo muy útiles y están sirviendo a los propios sindicatos para explorar alternativas a la huelga socialmente más aceptables que ella. Aunque se trata de un código muy singular, no me resisto a glosar la previsión contenida en el acuerdo italiano para la regulación de la huelga de los trabajadores que prestan sus servicios en helicópteros de socorro, que prevé, junto a la huelga tradicional, la huelga que el propio código Ilama virtual. ¿En qué consiste esta huelga? Pues básicamente en lo siguiente: si el sindicato opta por la huelga virtual durante el período de conflicto el servicio viene desempeñado regularmente, pero los trabajadores renuncian a su retribución y la empresa se obliga a abonar una iniciativa socialmente útil una suma igual a un múltiplo de los salarios dejados de percibir. Los trabajadores ejercitan la propia presión sobre la contraparte de manera directa, incidiendo de modo directo sobre su balance y no de modo indirecto tomando como rehenes a los usuarios. Es un modo de restaurar el viejo sentido de la huelga.

\section{EL CONFLICTO ANTE LOS NUEVOS MODELOS DE EMPRESA}

El segundo escenario en el que se producen cambios que son también, a la postre, cambios del conflicto es la empresa. Si el conflicto industrial tal y como lo concebimos encontró su natural caldo de cultivo en la gran empresa, en la que se forjaba "la conciencia de clase", los intensos procesos de reestructuración de los años ochenta y noventa han puesto en entredicho la viabilidad de la misma y han dado carta de naturaleza a una empresa magra, organizada en forma de red, que mediante el uso de nuevas tecnologías y el recurso intensivo del outssourcing ha alcanzado elevados niveles de productividad y demostrado gran capacidad de adaptación a los requerimientos del mercado.

Las estructuras productivas han adelgazado y se organizan de forma más flexible. El cambio, en efecto, opera en un doble sentido: de una parte, la estructura empresarial se trocea sustituyendo la gran empresa por una serie de empresas menores con las que se establecen intensas relaciones de colabora- 
ción económica; de otra, se ajusta la estructura productiva y de servicios de la empresa a aquellos segmentos del ciclo productivo en los que pueda aportarse alto valor añadido -el llamado core business- externalizando los demás.

En definitiva, tras un siglo básicamente caracterizado por una organización industrial basada en la integración vertical de la producción, las estrategias manageriales de hoy se dirigen a desintegrar los procesos productivos, distribuyendo cada una de sus fases entre unidades empresariales menores y sustituyendo en la medida de lo posible el trabajo subordinado por redes contractuales de naturaleza mercantil.

Las consecuencias de todo esto para la morfología del conflicto son evidentes. En la medida en que gran empresa pierde terreno y se sustituye por una miríada de pequeñas empresas, las dificultades para la agregación de intereses colectivos se multiplican y la formulación de plataformas reivindicativas con gran potencial movilizador se hace para el sindicato más difícil. El nuevo tejido productivo, y también la nueva realidad laboral como veremos, se revelan particularmente propicios para la proliferación de sindicatos autónomos, de oficio y de agregaciones de trabajadores para la defensa de intereses específicos, esto es, de formas de representación que pueden eludir la responsabilidad de valorar las consecuencias que de sus acciones se derivan para los intereses generales, elusión imposible para las grandes confederaciones sindicales.

La empresa debe, por otro lado, al tejer su red, prever y valorar su vulnerabilidad frente al conflicto laboral en cualquier segmento de la red que ha tupido. Un conflicto que para ella deja de ser laboral para pasar a ser mercantil, pues el conflicto en la red para ella no se traduce en el cese en el trabajo de los trabajadores a su servicio sino en incumplimientos contractuales de sus proveedores o contratistas que puede, eso sí, repercutir sobre su proceso productivo.

Pero quizá para la empresa matriz y también para los consumidores y usuarios en la medida en que la empresa preste servicios esenciales para la comunidad los mayores problemas pueden derivar de las medidas de conflicto adoptadas por los autónomos ligados a la empresa principal a través de contratos mercantiles, como, por ejemplo, un contrato de transporte. Es necesario establecer un marco jurídico para esas acciones colectivas, que no encajan en ningún caso en la huelga, pero que pueden ser lesivas para los servicios esenciales de la Comunidad y, desde luego, para la empresa, tanto o más que la huelga. Como es sabido, el régimen jurídico de la huelga es inaplicable a estas acciones colectivas que son también ceses colectivos de la prestación con finalidad reivindicativa. La huelga suspende las dos obligaciones fundamentales de la relación de trabajo -la de trabajar y la de abonar el salario- y no constituye en ningún caso un incumplimiento contractual, pues bien, tal cosa no puede decirse de la "huelga" de los autónomos, porque aquí la abstención colectiva sí constituye un incumplimiento contractual que puede dar lugar a responsabilidades civiles. 
Como todos ustedes saben, la Ley del Estatuto del Trabajo Autónomo, no ha querido abordar la cuestión y, por consiguiente, garantizar el mantenimiento de los servicios esenciales para la Comunidad, que son asimismo esenciales para la empresa en cuanto consumidor y usuario. En efecto, pese a que el art. 19 de la LETA reconoce a los trabajadores autónomos la titularidad del derecho a "ejercer la actividad colectiva de defensa de sus intereses profesionales", esta actividad es la prevista en la propia ley que no previene medidas de conflicto colectivo, esto es, lo que alguna asociación de autónomos Ilamó en sus propuestas durante el debate que precedió a la aprobación de la ley, el "paro reivincativo". Por tanto, pese a la novedosa norma, estamos donde estábamos y en este punto habrá que estar a las normas generales en materia de responsabilidad civil.

\section{El CONFLicto ANTE El NUEVO tRABAjO}

Pero si el sistema económico y la empresa han cambiado en los últimos decenios, no menos lo ha hecho el trabajo, que, escasísimo durante años, hoy recobra el pulso profundamente diversificado y se organiza, además, en la empresa de forma muy distinta.

Si la homogeneidad era la característica del trabajo "fordista", la heterogeneidad es la que define el trabajo "postfordista". El "universo del trabajo" es ahora mucho más rico que el que era y el sindicato hoy debe representar a trabajadores sometidos a estatutos jurídicos muy diversos y con intereses muy plurales: trabajadores contratados a jornada completa y por tiempo indeterminado; trabajadores contratados a tiempo parcial; trabajadores contratados a tiempo determinado, bien por la propia empresa o a través de una empresa de trabajo temporal; trabajadores temporales sujetos a un contrato formativo; trabajadores de empresas contratistas (multiservicios o no); trabajadores autónomos con intensa dependencia económica, socios trabajadores, desempleados, etc. la segmentación del trabajo que resulta de esta variedad hace decididamente de éste una realidad menos compacta y uniforme de lo que era en el pasado, dificultando consiguientemente la tarea de representación de intereses del sindicato. El sindicato está ahora Ilamado a articular y a conciliar toda esa diversidad de intereses.

Los cambios en la empresa y en los sistemas de organización del trabajo abundan, de otra parte, en la misma dirección, es decir, la de la diversificación de situaciones e intereses y la de la revalorización de las aportaciones y aspiraciones individuales. El trabajo cambia porque las profesiones ahora requeridas son las "profesiones del saber" que ejercen funciones más amplias, ricas y polivalentes, porque se fomenta el trabajo en grupo, porque se reduce la dependencia organizativa y jerárquica y porque se valora crecientemente la capacidad del trabajador de iniciativa, de adaptación a los cambios y de implicación en el 
proyecto empresarial. La exigencia empresarial de "calidad total" pasa necesariamente por un grado de implicación en la empresa del trabajador distinto del que era habitual en el taylor-fordismo, se requiere ahora un trabajador proactivo que comprometa su inteligencia al servicio del proyecto empresarial.

Como es obvio, estos cambios se están traduciendo, asimismo, en cambios en las relaciones de trabajo que, en última instancia, determinan cambios en el conflicto de trabajo. Por lo pronto, se han enriquecido sustancialmente los contenidos de la negociación colectiva, en la que cobran especial relevancia la regulación de los aspectos cualitativos del trabajo (clasificación profesional, retribución flexible, movilidad y modificación sustancial del tiempo de trabajo, ordenación del tiempo de trabajo y descanso, formación y promoción profesional, seguridad y salud laborales, protección del ambiente de trabajo, políticas de igualdad y no discriminación, incorporación de nuevas tecnologías, etc.), frente a los meramente cuantitativos -fijación de la jornada y el salario-, hasta el punto de que se habla del papel "organizacional" de la negociación colectiva, de la necesidad de que ésta asuma y desempeñe su función de adaptación del factor trabajo a la organización del trabajo y viceversa. En el elenco de materias que el Acuerdo Interconfederal para la Negociación Colectiva vigente contempla y respecto de las cuales establece criterios para la negociación colectiva estos aspectos cualitativos están bien presentes. Basta una lectura del índice del Acuerdo para percibirlo: formación y cualificación profesional, reestructuraciones, absentismos, igualdad de trato y oportunidades, seguridad y salud en el trabajo, estrés laboral, responsabilidad social de las empresas, etc.

Desde el punto de vista formal, ello se refleja en la propia factura del convenio colectivo, que junto a la regulación estricta de las concretas condiciones de trabajo abunda en la previsión de procedimientos de las mismas o su modificación, en línea con la filosofía de la Directiva 2002/14/CE, que establece un marco general relativo a la información y la consulta de los trabajadores de la Comunidad Europea. El convenio no es hoy sólo una norma que regula las condiciones de trabajo, sino también una norma procedimental para establecerlas, definirlas o modificarlas. Se "procedimentalizan" determinadas decisiones empresariales imponiendo procesos de negociación, garantizando así que en la adopción de las mismas los intereses de los trabajadores sean tenidos en cuenta.

Ejemplo paradigmático de esta "procedimentalización" son, por ejemplo, las medidas previstas para prevenir el acoso y la violencia en el lugar de trabajo en el "Acuerdo marco europeo", transcrito en anexo -curiosa implementación, por cierto- en la prórroga del AINC para el 2008. Se dice en el apartado 4 del Acuerdo: "Las empresas deben tener una declaración clara e inequívoca de que el acoso y la violencia no serán tolerados. Esta declaración especificará los 
procedimientos que se seguirán cuando se presenten los casos. Los procedimientos pueden incluir una etapa informal en la cual una persona de confianza del empresario y de los trabajadores esté disponible para dar consejo y ayuda (...). Un procedimiento adecuado se apoyará, de manera no limitativa en lo siguiente:

- Interesa a todas las partes proceder con la indiscreción necesaria para proteger la dignidad y la confidencialidad de todos. No se debe divulgar ninguna información a las partes implicadas en el caso.

- Las quejas deben ser investigadas y tratadas sin demoras indebidas.

- Todas las partes implicadas deben conseguir una audiencia imparcial y un tratamiento justo.

- Las quejas deben ser apoyadas por información detallada.

- Las acusaciones falsas no deben ser toleradas y pueden dar lugar a acciones disciplinarias. La ayuda externa puede ayudar.

- Si se comprueba que ha ocurrido acoso y violencia, se tomarán medidas apropiadas con relación a los autores. Esto puede significar acciones disciplinarias, incluso llegar al despido".

Los nuevos conflictos laborales tienen mucho que ver con esta nueva realidad del trabajo en la empresa. De una parte, y como consecuencia de esa mayor implicación personal que hoy exige el trabajo, la regulación adecuada del ambiente de trabajo en todos sus aspectos (derechos humanos en el trabajo, inexistencia de acoso moral o sexual, respeto al principio de igualdad y no discriminación, estrés en el trabajo, introducción de nuevas tecnologías, organización del trabajo en la empresa para la conciliación de la vida laboral y familiar, etc.) cobra cada día mayor relevancia y son cada vez más -como no podía ser de otra forma- fuentes de conflicto. El trabajador es cada día más sensible a las condiciones del entorno en el que presta su trabajo, hoy éstas para el trabajador importan si no más tanto como la retribución que percibe por el trabajo. En la regulación de estos aspectos, la ley, como probablemente no podría ser de otro modo, acostumbra a ser parca, no pasando de establecer normas semielaboradas, enunciadas como principios, cuya aplicación práctica requiere de la adaptación a la singularidad de la empresa y de su organización del trabajo, cometido que obviamente recae sobre la negociación colectiva. Pues bien, tanto la aplicación, a falta de desarrollo convencional, de estos nuevos "principios" generales, como la aplicación de la regulación convencional van a ser -están siendo ya-fuentes de conflicto, unos conflictos que, en puridad, puesto que abordan cuestiones de organización del trabajo en la empresa, son conflictos de intereses y no jurídicos. Sería bueno desjudicializar estos conflictos y que fueran procedimientos extrajudiciales de negociación, más apegados a la realidad de la empresa, los que solventarán propiciando acuerdos razonables estas contiendas. 
Les pondré sendos ejemplos de lo que digo, derivados ambos de la nueva Ley de Igualdad, que, como ha sido puesto de relieve, es una ley llamada a producir importantes efectos a medio y largo plazo en nuestro sistema de relaciones laborales.

El primero deriva del nuevo párrafo 8 añadido por la Ley de Igualdad al art. 34 del Estatuto de los Trabajadores, precepto conforme al cual: "El trabajador tendrá derecho a adaptar la duración y distribución de la jornada de trabajo para hacer efectivo su derecho a la conciliación de la vida personal, familiar y laboral en los términos que se establezcan en la negociación colectiva o en el acuerdo a que llegue con el empresario respetando, en su caso, lo previsto en aquélla". Se trata de un precepto importante, pues concreta una vertiente del derecho a la conciliación de la vida personal y familiar, pero a nadie se le escapa que el ejercicio del derecho que reconoce va a ser problemático y conflictual, en la medida en que incide sobre las prerrogativas empresariales de organización del trabajo en la empresa, con las que también habrá que conciliarse. Pues bien, dada la dimensión "organizacional" del asunto y de los conflictos que del mismo se deriven, parece sensato que de estos conflictos conozcan los procedimientos extrajudiciales de solución y no los órganos judiciales, que siempre resuelven desde el presupuesto de la unidad de solución justa y ello por más que la nueva DA $17^{\mathrm{a}}$ del ET remita a la jurisdicción social y al art. 138 bis para la resolución de estos conflictos.

Algo parecido puede predicarse de los conflictos que sin duda suscitará la elaboración y aplicación de los Ilamados planes de igualdad, cuya negociación y aplicación está prevista en la ley para las empresas de más de doscientos cincuenta trabajadores y cuando lo exija el convenio colectivo aplicable. Estos planes, que deben constituir un conjunto ordenado de medidas, adoptadas después del diagnóstico de la situación, deben fijar "Ios concretos objetivos de igualdad a alcanzar, las estrategias y prácticas a adoptar para su consecución, así como el establecimiento de sistemas eficaces de seguimiento y evaluación de los objetivos fijados" (art. 46 LOI). La prórroga del AINC para el 2008, en línea por cierto con la tendencia apuntada más arriba, instruye convenientemente a los negociadores sobre cómo implementar estas previsiones legales. Pues bien, a poco que estos planes sean mínimamente incisivos, su interpretación y aplicación será fuente de conflictos que habría que intentar desjudicializar por las mismas razones expuestas más arriba. Sería conveniente que en los propios planes y en la negociación colectiva que los incorpore o regule se prevea que estos eventuales conflictos se resuelvan a través de sistemas autónomos de composición de conflictos, pues insisto creo que es éste el ámbito más adecuado para solventarlos.

El carácter "gestional" u "organizacional" del nuevo convenio va a hacer que los conflictos que tienen por objeto la administración del mismo proliferen, 
entendiendo por administración no sólo la interpretación del convenio, sino también la aplicación de los procedimientos que en el mismo se pacten para la determinación o modificación de las condiciones pactadas. No estoy refiriéndome a la modificación del convenio, sino a la aplicación estricta del mismo que, sin embargo, incorpora mecanismos de carácter "gestional" que consisten en la adaptación del trabajo a las necesidades de la empresa y el mercado. Como ustedes saben, nuestra jurisprudencia distingue netamente lo que es modificación del convenio de lo que es su interpretación y limita enormemente las posibilidades de actuación novatorias de las Comisiones Paritarias. Yo creo que en la medida en la que el convenio colectivo avance en su dimensión "organizacional" las fronteras entre la modificación y la interpretación del convenio se difuminarán y que nuestra jurisprudencia debiera ser más laxa al enjuiciar la labor de las Comisiones Paritarias. Sea como fuere, lo cierto es que los conflictos derivados de la administración del convenio, entendida tal y como lo he hecho, deben ser un filón para los procedimientos de solución autónoma de conflictos, pues al cabo estos son un modo de recrear la autonomía colectiva que es fundamento del convenio y, si me apuran, de un sistema democrático de relaciones laborales. 
